
allemande

\title{
Représentation et fonction de la catastrophe dans les thrillers climatiques
}

Prophezeiung (2011) de Sven Böttcher et Sturm (2017) de Uwe Laub

\section{Olivier Hanse}

\section{(2) OpenEdition}

\section{Journals}

Édition électronique

URL : https://journals.openedition.org/allemagne/2288

DOI : $10.4000 /$ allemagne.2288

ISSN : 2605-7913

\section{Éditeur}

Société d'études allemandes

Édition imprimée

Date de publication : 1 juillet 2020

Pagination : 159-176

ISSN : 0035-0974

Référence électronique

Olivier Hanse, «Représentation et fonction de la catastrophe dans les thrillers climatiques », Revue d'Allemagne et des pays de langue allemande [En ligne], 52-1 | 2020, mis en ligne le 06 juillet 2021, consulté le 19 février 2022. URL : http://journals.openedition.org/allemagne/2288 ; DOI : https:// doi.org/10.4000/allemagne.2288 


\section{Représentation et fonction de la catastrophe dans les thrillers climatiques} Prophezeiung (2011) de Sven Böttcher et Sturm (2017) de Uwe Laub

\section{- Olivier Hanse*}

Si le thriller a le plus souvent tendance à être considéré comme un genre mineur et avant tout divertissant, la question peut se poser dans des termes quelque peu différents au sujet de sa version écologique, à l'heure où l'ampleur dramatique des dégradations environnementales causées par l'homme justifie pour une majorité de scientifiques la reconnaissance du concept transdisciplinaire d'Anthropocène en tant que nouvelle ère géologique dominée par les activités humaines ${ }^{(1)}$. Le thriller écologique, qui connaît depuis son émergence dans les années 90 une percée spectaculaire, et dont le «thriller climatique» ne constitue qu'une variante particulièrement actuelle, occupe une place à la fois centrale et singulière parmi ce qu'il convient, avec la comparatiste Evi Zemanek, d'appeler les «genres écologiques » ${ }^{(2)}$, place que notre première partie tentera de préciser.

Les deux ouvrages étudiés en leur qualité d'exemples particulièrement éloquents de cette vogue littéraire ont pour principaux points communs d'être des succès de librairie relativement récents, puisqu'ils ont tous deux été publiés durant la deuxième décennie du $\mathrm{XXI}^{\mathrm{e}}$ siècle, d'user de techniques narratives propres au thriller et au roman à suspense, et de mettre en scène, par l'écriture romanesque, des catastrophes climatiques dignes des plus grands blockbusters hollywoodiens. Rappelons notamment que le film catastrophe de Roland Emmerich The day after tomorrow, véritable modèle du genre dont l'action est également fondée sur l'hypothèse d'un dérèglement climatique brutal et d'ampleur planétaire, est sorti en 2004 sur les écrans. Du point de vue du contexte historique, on

* Maître de conférences au Centre d'études germaniques interculturelles de Lorraine (CEGIL), Université de Lorraine - site de Metz.

1 Gabriele DüRBECK, «Das Anthropozän in geistes- und kulturwissenschaftlicher Perspektive», in: Gabriele Dürвеск, Urte Sтовве (éd.), Ecocriticism. Eine Einführung, Cologne, Böhlau, 2015, p. 107-119.

2 Evi Zемалек (éd.), Ökologische Genres, Göttingen, Vandenhoeck \& Ruprecht, 2018. 
peut en outre souligner que la thématique retenue est en quelque sorte conditionnée par les événements qui secouent la planète et les pronostics proclamés par de nombreux experts. Lors de la sortie du bestseller de Frank Schätzing Der Schwarm (2004), supposé avoir fait acquérir ses lettres de noblesse au thriller écologique de langue allemande ${ }^{(3)}$, les images du tsunami meurtrier de 2004 en Asie du Sud-Est sont encore bien présentes dans les esprits, tandis que la catastrophe nucléaire de Fukushima en 2011, qui faisait suite à l'un des plus importants séismes de l'histoire du Japon puis au ravage de $600 \mathrm{~km}$ de côtes par un tsunami consécutif à la secousse sismique, a servi de contexte à la parution de Prophezeiung (2011) de Sven Böttcher ${ }^{(4)}$. Entre ces deux événements, l'obtention conjointe du prix Nobel de la Paix en 2007 par l'ancien vice-président des États-Unis Al Gore, porteur d'une spectaculaire campagne de sensibilisation sur le réchauffement planétaire, et par le GIEC (Groupe d'experts intergouvernemental sur l'évolution du climat, en anglais IPCC), dont le troisième rapport d'évaluation de 2001 a considérablement contribué à faire entrer la thématique du changement climatique d'origine humaine dans les débats politiques et médiatiques, a entre autres fait écho à la diffusion en 2006 du documentaire américain An Inconvenient Truth de Davis Guggenheim qui, sur fond d'images spectaculaires, présentait les effets dramatiques du réchauffement de la planète et développait en particulier un certain nombre de scénarios-catastrophes comme le risque d'effondrement d'un inlandsis groenlandais, susceptible d'élever le niveau des océans de près de 6 mètres, de provoquer des inondations d'une ampleur inédite ainsi que l'errance de plusieurs dizaines de millions de réfugiés climatiques. Sorti en 2017, Sturm d'Uwe Laub ${ }^{(5)}$ est encore clairement sous l'influence de l'Accord de Paris de décembre 2015 et de la prise de conscience croissante du monde occidental face à l'impact des gaz à effet de serre et à l'augmentation probable des événements climatiques extrêmes dans les prochaines décennies.

À travers l'étude de la représentation des catastrophes climatiques dans les deux romans retenus, il s'agira avant tout d'en évaluer la fonction tout en posant la question de son éventuelle portée écologique et politique. Les désastres qui sont au centre de ces romans sont-ils conçus comme de véritables repoussoirs, des évolutions possibles de notre monde qu'il s'agirait par tous les moyens d'éviter, ou bien peuvent-ils être perçus comme étant primairement au service d'autres aspirations propres à nos sociétés?

\section{Un genre hybride alliant divertissement et réflexion critique}

Le schéma fondamental du thriller écologique, dont les principaux ouvrages présentent une dimension cinématographique assez prononcée, peut être décrit avec la germaniste Gabriele Dürbeck de la manière suivante:

«En général, il présente une catastrophe environnementale locale ou globale, qui souvent menace de provoquer l'extinction de toute l'humanité mais qui, grâce à une action

3 «Mit Frank Schätzings Roman Der Schwarm wurde 2004 der deutschsprachige Thriller wieder salonfähig gemacht. » Katrin SchneIDER-ÖzBEK, «Der Ökothriller. Zur Genese eines neuen Genres an der Schnittstelle von Thriller und ökologischem Narrativ», in: E. ZEMAnEK (éd.), Ökologische Genres (note 2), p. 229-246, ici p. 229.

4 Sven Böttcher, Prophezeiung, Cologne, Kiepenheuer \& Witsch, 2011.

5 Uwe Laub, Sturm, Munich, Heyne, 2018 (2 éd.). 
collective souvent hérö̈que, parvient in extremis à être évitée. Dans ce cadre, la crise environnementale et climatique est la plupart du temps représentée comme la conséquence de la domination anthropocentrique de la nature, d'une idéologie du progrès de type rationaliste et de l'exploitation économique et technique des ressources naturelles. Tout en faisant apparaître les dessous de la crise et en révélant de possibles alternatives, le thriller écologique met en œuvre des stratégies de représentation critiques et réflexives propres à la popularisation scientifique, et intègre celles-ci dans une histoire pleine de suspense. Ainsi, le genre du thriller écologique se caractérise par une tension entre sa volonté d’informer et celle de divertir» ${ }^{(6)}$.

Comme l'a très clairement fait ressortir Dürbeck, le thriller écologique est avant tout un genre hybride qui emprunte ses éléments constitutifs à au moins trois autres formes d'expression littéraire ou cinématographique ${ }^{(7)}$. La première est, de toute évidence, la science-fiction qui, née dans la seconde moitié du XIX ${ }^{\mathrm{e}}$ siècle, apparaît comme le genre par excellence de l'anticipation des évolutions scientifiques et sociétales et semble particulièrement appropriée pour engager une réflexion critique sur les progrès technologiques et les travers de notre civilisation. Nombre d'œuvres de ce genre sont des dystopies, ou des contre-utopies, qui visent à penser jusqu'au bout les conséquences probables de certaines évolutions contemporaines jusqu'à aboutir à une véritable vision de cauchemar, la représentation de mondes terrifiants permettant une prise de conscience sur les prolongements possibles de certaines idéologies ou pratiques présentes à notre époque. Parmi les classiques du roman d'anticipation dystopique, apparenté à la science-fiction, de nombreux ouvrages constituent des références incontournables dont l'influence sur certains thrillers écologiques ne peut être ignorée. Le Meilleur des mondes (1931) d'Aldous Huxley, 1984 (1949) de George Orwell, La Planète des singes (1963) de Pierre Boulle et Soleil Vert (1966) de Harry Harrison, ouvrages porteurs d'une futurologie très pessimiste et qui ont tous fait l'objet d'adaptations cinématographiques ou télévisuelles, comptent de toute évidence parmi les plus célèbres références.

Ainsi, pour ne donner qu'un seul exemple, le roman de Dirk C. Fleck intitulé Go! Die Ökodiktatur $(1993)^{(8)}$, qui a reçu en 1994 le prix allemand de la science-fiction et se voit fréquemment décrit comme un des prototypes allemands du thriller écologique, situe son action en l'an 2040, dans un monde meurtri par une détérioration radicale des conditions environnementales, et dépeint, à la manière d'Aldous Huxley et de George Orwell, les diverses facettes d'un univers cauchemardesque à la fois proche du nôtre et profondément différent. Dans le système totalitaire qui s'est mis en place au sein des anciennes nations industrielles occidentales pour éviter le collapse irréversible du vivant sur Terre, toute forme de voyage touristique et de construction immobilière est interdite, l'État a aboli la presse, contrôle la répartition de l'habitat, l'alimentation (obligatoirement végétarienne) et concentre dans des camps les malades contagieux ainsi que tous ceux qui, d'une manière ou d'une autre, ont enfreint un des douze articles de la Loi fondamentale supposée avant tout asseoir la dignité imprescriptible

6 Gabriele Dürвеск, «Ökothriller», in: E. Zемалек (éd.), Ökologische Genres (note 2), p. 245-257, ici p. 245 (notre traduction).

7 Ibid., p. 246-250.

8 Dirk C. FleCK, Go! Die Ökodiktatur. Erst die Erde, dann der Mensch, Winnert, p. machinery, 2013. 
de la planète ${ }^{(9)}$. L'anticipation de la déchéance de nos démocraties, de la fin des libertés individuelles, présentée par Fleck dans son roman ainsi que dans diverses prises de position ultérieures comme la conséquence ultime et de plus en plus inévitable d'une catastrophe trop longtemps ignorée ${ }^{(10)}$, relie ce texte de manière perceptible à certains grands classiques de la science-fiction.

Notons de surcroît qu'une certaine tendance de la part des auteurs contemporains de science-fiction à placer l'action de leurs romans dans un futur de plus en plus proche, notamment dans le but de mobiliser un certain lectorat en faveur de la transformation écologique de nos sociétés, a provoqué depuis un peu plus de dix ans l'émergence de la «cli-fi» (ou climate fiction), terme lancé en 2008 par l'écrivain et blogueur américain Dan Bloom pour désigner toute une série d'ouvrages aux accents eschatologiques visant à "éveiller les consciences à l'aide de récits apocalyptiques » ${ }^{(11)}$. Ce genre émergent ${ }^{(12)}$, dont Gold Fame Citrus (2015) de la jeune auteure californienne Claire Vaye Watkins est actuellement un des succès les plus remarqués sur la scène littéraire internationale, semble avoir connu un développement à la fois plus précoce et plus marqué aux États-Unis, pays où, comme le souligne très justement Claire Perrin, "des personnalités climatosceptiques occupent [...] les plus hautes fonctions», ce qui renforce la légitimité de tels récits à véritablement «faire œuvre de résistance» ${ }^{(13)}$.

La deuxième tradition littéraire dans laquelle s'inscrivent les auteurs de thrillers écologiques est de toute évidence celle du thriller, marqué notamment par la mise en œuvre de stratégies narratives visant à provoquer chez le lecteur ou le spectateur une tension, excitation ou appréhension capable de le tenir en haleine jusqu'au dénouement de l'intrigue. Le suspense, l'anticipation d'événements dramatiques, la présence d'une énigme qui se noue et se précise au fur et à mesure que progresse la narration, l'introduction de scènes de poursuite et de combat, voire d'actions violentes, qui invitent à

9 Ibid., p. 15.

10 Lire notamment son plaidoyer assez violent en faveur du régime qu'il a imaginé, une conférence prononcée successivement en 1994 dans les universités de Innsbruck, Hambourg et Bonn: «Eine Ökodiktatur, wie ich sie notgedrungen kommen sehe, ist nicht mit herkömmlichen moralischen Maßstäben zu messen. Moral taugt nichts angesichts des kollektiven Untergangs. An dieser Stelle wird Politik zum Notwehrreflex. Die freie Gesellschaft hat demnächst ausgedient. Unsere Kinder drohen zu Überlebensmonstern zu mutieren, die es durch straffe Herrschaftsstrukturen unter Kontrolle zu halten gilt.» Ibid., p. 193-209, ici p. 194. La position ambiguë de Dirk C. Fleck sur ce point fait l'objet d'une mise en perspective plus large des discours sur la dictature écologique dans: Daniel STEIGER, Imaginierte Ökodiktaturen. Interdisziplinäre Perspektiven auf ein gesellschaftspolitisches Konstrukt, Siegen, universi, 2015, p. 51-62.

11 Claire Perrin, "La "cli-fi", quand la science-fiction rencontre l'écologie», Slate.fr (en ligne), 27 décembre 2018, www.slate.fr/story/171624/litterature-cli-fi-climate-science-fiction-changementclimatique (consulté le 29.12.2019).

12 Rio Fernandes, "The Subfield That is Changing the Landscape of Literary Studies", in: The Chronicle of Higher Education, 21 mars 2016, www.chronicle.com/article/The-Subfield-That-IsChanging/235776 (consulté le 01.01.2020).

13 C. Perrin, «La "cli-fi", quand la science-fiction rencontre l'écologie» (note 11). Claire Perrin résume également en ces termes la polémique suscitée par le catastrophisme de ce genre: "Ces accents eschatologiques exposent [...] ce genre littéraire à la critique de la frange plus modérée des écologistes: représenter le réchauffement climatique comme un phénomène apocalyptique revient à se couper l'herbe sous le pied en risquant de désintéresser le public de la question, convaincu par ces œuvres qu'il n'y a plus rien à faire.» 
craindre pour la survie de l'héroïne ou du héros, constituent autant d'éléments caractéristiques de cette forme de littérature essentiellement tournée vers le divertissement (même si le genre n'exclut pas la réflexion) et au sein de laquelle la majorité des personnages se trouve globalement réduite à une palette stéréotypée de caractéristiques liée à leur sexe, à leur condition sociale et à leur profession.

«Au centre de nombreux thrillers écologiques se trouvent des scientifiques et des journalistes (scientifiques) des deux sexes. Les protagonistes (héroïques) agissent le plus souvent conformément à des schémas manichéens dans le cadre de narrations liées à des services secrets, des conjurations, de l'espionnage ou de la guerre froide, et sont personnellement mis en danger de mort par les représentants sans scrupules d'intérêts économiques, technologiques ou militaires d'une ou plusieurs nations (souvent occidentales). Mais grâce au soutien de sympathisants qui apparaissent de manière soudaine ou de groupes qui sympathisent avec eux et se sont formés pour combattre la destruction environnementale, ils trouvent un moyen d'empêcher l'extinction qui menace l'humanité ou d'en réduire le danger ${ }^{(14)}$.

Enfin, la transmission de connaissances et la vulgarisation scientifique jouent un rôle tellement important dans le genre émergent du thriller écologique qu'il a fréquemment été mis en rapport avec le documentaire-fiction, un genre hybride lui aussi, qui s'est considérablement développé au cinéma et à la télévision depuis une vingtaine d'années et ne doit pas être confondu avec le «faux documentaire» (encore appelé documenteur, ou en anglais pseudo-documentary), lequel a pour principe de présenter de manière plus ou moins ludique des faits parfaitement fictifs sous la forme d'un documentaire, notamment dans le but de défendre fictivement des thèses farfelues, d'embrouiller volontairement le spectateur ou de jouer avec l'«éthique documentaire» en vue de «questionner notre rapport à l'image» ou au texte ${ }^{(15)}$. Dans le documentaire-fiction, la fiction et la non-fiction sont certes inextricablement liées ${ }^{(16)}$, mais la première est avant tout mise au service d'un objectif à la fois esthétique et pédagogique, puisqu'il s'agit d'abord de donner une forme attrayante à un contenu historique ou scientifique pour y intéresser un public plus large et, ainsi, réconcilier le documentaire avec l'audimat et l'«impératif de divertissement ${ }^{(17)}$. De par sa volonté de transmettre des connaissances scientifiques sérieuses et aisément vérifiables tout en les insérant dans une histoire palpitante au sein de laquelle réalité et fiction finissent par se confondre, le thriller

14 G. DÜRBECK, «Ökothriller» (note 6), p. 250 (notre traduction).

15 Joris Laquittant, "Toute la vérité sur le documenteur», Fais pas genre (en ligne), 20 juillet 2017, faispasgenre.com/2017/07/toute-verite-documenteur/ (consulté le 04.01.2020). Joris Laquittant mentionne quelques exemples de détournements ludiques: «[L]e cinéma n’a pas été inventé par les Frères Lumière mais par un Néo-Zélandais du nom de Colin McKenzie (Forgotten Silver de Peter Jackson, 1995), les Américains n’ont pas marché sur la Lune et c'est Stanley Kubrick qui a réalisé en studio les images de l'alunissage (Opération Lune de William Karel, 2002), Joaquin Phoenix a arrêté sa carrière d'acteur en pleine gloire pour se lancer dans le rap (I'm Still Here de Casey Affleck, 2010) ou enfin, une vaste entreprise complotiste ourdie par Hollywood empêche le cinéma européen d'exister (Hitler à Hollywood de Maria de Medeiros, 2011).»

16 Cf. Philipp Blum, «Doku-Fiktionen. Filme auf der Grenze zwischen Fiktionen und Non-Fiktionen als ästhetische Interventionen der Gattungslogik», MEDIENwissenschaft, 2 (2013), p. 130-144.

17 François GARçON, «Le documentaire historique au péril du "docufiction" », Vingtième Siècle. Revue d'histoire, $\mathrm{n}^{\circ} 88,4$ (2005), p. 95-108, ici p. 104. Un des plus grands succès français à la télévision fut le téléfilm de Jacques Malaterre L'Odyssée de l'espèce (2003) qui, dès sa première diffusion, fut regardé sur France 3 par près de 9 millions de téléspectateurs. 
écologique est clairement influencé par le documentaire-fiction. Parmi les exemples les plus manifestes d'une telle proximité, on trouve, aux côtés du célèbre roman d'Andreas Eschbach Ausgebrannt (2007) sur la fin de l'ère du pétrole, l'ouvrage déjà mentionné de Frank Schätzing Der Schwarm (2004), dont l'auteur, féru de géophysique et de microbiologie, publia deux ans après sa parution un livre de vulgarisation scientifique sur les mystères des profondeurs marines, Nachrichten aus einem unbekannten Universum (2006), qui compile des informations tirées de nombreux articles scientifiques n'ayant pas trouvé directement leur place au sein du roman, avant de participer activement à sa transformation par la chaîne ZDF en un documentaire à gros budget et en trois parties, Terra X - Universum der Ozeane (2010), dans lequel il apparaît en tant que présentateur. Le roman lui-même débute par la mise en scène d'une série d'incidents et de catastrophes naturelles tout d'abord incompréhensibles, qui ont lieu de manière quasi concomitante en divers endroits du globe, et aboutit à la reconnaissance de l' "yrr», un conglomérat d'organismes unicellulaires formant ensemble une intelligence supérieure à celle de l'humanité et qui se dresse soudain contre cette dernière pour se venger de son exploitation féroce de la nature et des océans. L'auteur entrecoupe le déroulement de son intrigue par des passages explicatifs longs et ardus qui apportent des précisions sur les courants marins, les bactéries, les plateformes pétrolières, la vie des baleines, le plateau continental ou encore les différentes techniques de plongée et l'histoire de la planète. Fondés sur une documentation scientifique sérieuse et abondante, ces passages ralentissent considérablement la progression de l'action et contribuent au positionnement ambigu de cet ouvrage de plus de mille pages entre documentaire et fiction.

À la jonction entre science-fiction, thriller et documentaire-fiction, le thriller écologique permet ainsi de rendre accessible à un large public des connaissances scientifiques plus ou moins pointues tout en les intégrant à une fiction captivante qui invite son lecteur à s'interroger sur son avenir proche ainsi que sur les structures du monde qui l'entoure. De la sorte, par-delà le suspense qu'il entretient et sa finalité avant tout divertissante, ce genre renferme en lui-même un potentiel critique, même s'il ne bascule que très rarement dans l'engagement politique. Visant entre autres à combattre l'ignorance des enjeux environnementaux, il confronte le grand public à de possibles évolutions susceptibles de nourrir sa réflexion et d'orienter ses choix. Analyser le traitement de la catastrophe dans deux thrillers climatiques allemands de la dernière décennie doit permettre de préciser l'apport du genre au discours écologique ainsi que la fonction du catastrophisme porté par de tels ouvrages.

\section{La catastrophe climatique entre pluralité des discours et théâtre de l'action dramatique}

Sven Böttcher, qui s'était jusque dans les années 2010 essentiellement fait connaître en tant que scénariste pour la télévision, traducteur et auteur de polars, place la catastrophe climatique, caractérisée par un bouleversement brutal du cycle hydrologique de la planète, au centre de son roman. La «prophétie» annoncée par le titre même de l'ouvrage renvoie au fait qu'un dérèglement d'ampleur planétaire domine la première partie du thriller sous la forme d'une "prévision de la fin du monde » ${ }^{(18)}$ établie grâce 
au programme informatique Prometheus. Ce dernier, un projet météorologique strictement confidentiel élaboré sur l'île de La Palma par une obscure institution nommée IICO (International Institute for Climate Observation), s'avère capable de prédire le temps en tout point du globe avec une précision jusque-là inconnue. Après s'être introduite dans le système de manière illicite, la climatologue et principale héroïne nouvellement arrivée à l'IICO, Mavie Heller, vérifie la concordance impressionnante entre les prévisions du programme, manifestement capable de prendre en considération des données longtemps restées inaccessibles, et les valeurs mesurées lors des mois précédents, sans que le lecteur apprenne le fondement exact de ces calculs. Elle découvre que l'institut est en train de cacher à l'humanité un bouleversement climatique imminent: des températures printanières au mois de janvier dans l'hémisphère nord, accompagnées de précipitations ininterrompues pendant de nombreux mois, tandis que les régions proches de l'équateur seraient touchées par une chaleur et une sécheresse exceptionnelles, le tout étant supposé provoquer des CD (Collateral Damages) de plusieurs centaines de millions de victimes $^{(19)}$ ainsi que l'exode de pays entiers et une catastrophe à la fois logistique et humanitaire sans précédent. Malgré le renvoi de l'héroïne pour faute professionnelle, le doute subsiste pendant un certain temps sur la véracité à moyen et long terme de la prévision que l'ami et ancien professeur de Mavie, Fritz Eisele, tente dans un premier temps de lui présenter comme une simple «simulation ${ }^{(20)}$.

À partir du moment où la catastrophe devient vérifiable, la prophétie de l'application Prometheus engendre une pluralité de discours, qui se retrouvent successivement relativisés voire décrédibilisés, si bien qu'aucun de leurs porteurs ne semble pouvoir être reconnu comme le représentant d'une position soutenue par l'auteur.

Le premier discours est celui des climatologues, représentés par le personnage de Thilo Beck, un collègue de Mavie à l'IICO qui, suite au meurtre de la journaliste Helen, devient un des principaux alliés de l'hérö̈ne dans sa quête de vérité. Lors de leur rencontre à l'hôtel New York de Rotterdam, celui-ci livre en quelque sorte le point de vue de l'expert sur le dérèglement climatique en cours et la probable véracité des prévisions de Prometheus. Le scientifique compare le phénomène à une sorte d' «AVC de la planète» ${ }^{(21)}$ provoqué en premier lieu par un phénomène naturel, à savoir une augmentation soudaine du rayonnement solaire provoquant une modification spectaculaire de la répartition de l'eau et du cycle hydrologique à l'échelle planétaire ${ }^{(22)}$. Selon Beck, la production de $\mathrm{CO}_{2}$ d'origine humaine n'est de toute évidence pas la cause primaire du réchauffement climatique en cours et de la catastrophe qu'il engendre, mais elle en constitue néanmoins un élément aggravant:

«Lors des dernières attaques, ce patient [la planète] ne portait pas encore une grosse veste de gaz à effet de serre lors de ses marathons forcés. Le climat fait certes ce qu'il veut, le climat se comporte comme un ivrogne qui traverse la zone piétonne en trébuchant, mais il n'est pas très malin d'énerver l'ivrogne par-dessus le marché. Or c'est ce que nous avons fait, pendant des décennies. Et maintenant, il est trop tard pour mettre encore un casque » ${ }^{(23)}$.

19 Ibid., p. 50-57.

20 Ibid., p. 62.

21 Ibid., p. 155.

22 Ibid., p. 156.

23 Ibid., p. 159. 
Bien qu'il dispose des clés nécessaires à la compréhension du phénomène, le discours de l'expert se caractérise par un refus de prendre des responsabilités et une tendance à l'immobilisme. Alors que les données se vérifient jour après jour, Thilo Beck demeure prisonnier de la précision du discours scientifique en refusant notamment de tirer des concordances « arithmétiques » observées la conclusion définitive que «la prévision est juste» ${ }^{(24)}$, puis en insistant sur le fait que l'incapacité du système à chiffrer de manière précise les «modifications de comportement humain dans des situations de crise» ${ }^{(25)}$ contraint à prendre avec prudence le pronostic en termes de victimes. Cette attitude provoque la sidération de l'héroïne: «Elle secoua la tête avec perplexité. Mais tout ceci était sans importance. Même si Prometheus se trompait statistiquement parlant de 50 terribles pourcents, sa prévision était une catastrophe et coûterait la vie à 400 millions de personnes» ${ }^{(26)}$. Interrogé sur son inaction depuis qu'il a accès à ces informations, Beck se cache derrière la décision de l'institut et insiste sur la panique que causerait l'évacuation d'un bon milliard d'individus et les conséquences dramatiques qu'une telle tentative pourrait avoir, avant de camper sur son statut de chercheur, non habilité à prendre l'initiative d'une telle annonce: «Je ne suis pas le sauveur du monde. Je suis un scientifique. Je fais ce que j'ai à faire. Et si mon chef et ses chefs pensent qu'il est tout d'abord encore nécessaire de lancer une série de tests, je ne suis pas contre, mais je suis pour. Résolument » ${ }^{(27)}$.

Le refus du scientifique d'endosser la responsabilité de l'action au nom d'un doute résiduel appelant une vérification préalable apparaît comme une sorte de leitmotiv dans le roman et permet d'opposer l'immobilisme de Beck à l'action permanente, souvent prématurée et inconsidérée, des activistes écologistes, au sein desquels est engagée Paulina, la sœur du climatologue. Cette dernière, défendant face à son frère convalescent la nécessité de faire éclater la vérité au grand jour pour permettre aux potentielles victimes de prendre leur destin en main, condamne cet empêtrement dans l'inaction en des termes crus et sévères qui semblent invalider le discours du climatologue, au moins jusqu'à la découverte, vers la fin du roman, que la prévision est effectivement erronée:

«C'est ça ta maladie, Thilo, tu as le cul collé sur ton p... de fauteuil. Tu as toujours voulu tout étudier en détail et tout vérifier 5000 fois, et de préférence encore tout recalculer. Et entre-temps, dehors, la vie continue et le temps que tu aies enfin terminé ton calcul, tout a déjà eu lieu depuis longtemps - et tes calculs à la con ne servent plus à rien " ${ }^{(28)}$.

Le deuxième discours sur le dérèglement climatique est celui de Leland Milett, lauréat d'un double prix Nobel ayant pour diverses raisons obscures longtemps disparu de la sphère publique et que Mavie, son père et son compagnon Philipp parviennent à convaincre de mettre sa notoriété au service de l'annonce de la catastrophe imminente. Tout d'abord réticent, l'homme de lettres se sent progressivement investi d'une mission inespérée et finit par endosser pleinement la fonction ressentie de «sauveur de 
l'humanité », seul capable d'éviter le massacre en train de se profiler. Dans son intervention face à la presse internationale organisée dans sa maison du sud de la France et retransmise par de nombreuses chaînes de télévision, Milett prend en premier lieu ses distances vis-à-vis des hommes politiques, accusés d'avoir préféré ignorer le désastre le plus longtemps possible, comme vis-à-vis des «prétendus idéalistes qui, sous des noms incongrus, ne poursuivent pas d'autre but que l'anéantissement du monde et de tout ce qui nous est cher " ${ }^{(29)}$, désignant ainsi le commando des Gaia Camps de Diego Garcia, le compagnon et l'idole de la sœur de Thilo Beck, alors que ceux-ci viennent tout juste de poster sur Internet et les réseaux sociaux un clip vidéo divulguant la catastrophe en cours. Par opposition à ces deux groupes jugés irresponsables, Milett assume d'une part son rôle de "messager apportant la mauvaise nouvelle ${ }^{\left({ }^{(0)}\right.}$, qui confirme la véracité de l'apocalypse annoncée ainsi que son fondement scientifique, mais il se présente en outre comme un porteur d'espoir et le possible coordinateur d'une action conjointe de toutes les nations, ce qui n'est pas sans rappeler la bataille ultime du film Independance Day (1996) de Roland Emmerich, un assaut de la dernière chance pour la survie de l'espèce humaine, à la différence que l'ennemi à combattre n'est pas ici une colonie d'extraterrestres venus asservir les hommes et piller les ressources de la planète, mais la nature comme entité globale opposée à l'homme:

«L'avenir de l'humanité dépend de notre solidarité, de notre volonté de changement et de notre action coordonnée. La nature, que les Romantiques parmi nous appellent Mère ou Gaia, prévoit de nous exterminer par milliards. Celui qui, dans un cas pareil, ne résiste pas, celui qui ne dit pas que les hommes passent avant la nature, celui-là pense et agit de manière inhumaine. Qui, sur cette voie, n'est pas de notre côté, du côté des hommes, est notre opposant et notre ennemi. Jusqu'à ce que la crise soit surmontée, le mot d'ordre ne sera pas: nous devons protéger la nature. Il sera au contraire: nous devons et nous allons nous protéger nous-mêmes contre la nature, nous et toute l'humanité ${ }^{(31)}$.

Outre la référence évidente au discours légendaire de George Bush à la suite des attentats du 11 septembre ("You are either with us or against us»), la position défendue par Milett est immédiatement invalidée par la narration, qui présente d'emblée le lauréat du prix Nobel comme un personnage fondamentalement comique à tendance mégalomane, sorte de "mélange entre un secrétaire général de l'ONU et Richard Branson » ${ }^{(32)}$ (ou bien Moïse divisant la mer Rouge, mais en bien plus beau, compte tenu du travail irréprochable de sa coiffeuse attitrée!) ${ }^{(33)}$. Totalement exagérée, la mise en scène du discours, dont on pourrait croire qu'il est tenu en direct de la Maison Blanche ${ }^{(34)}$ par un homme qui brigue «le cumul de la présidence des États-Unis et de la papauté sur sa propre personne» ${ }^{(35)}$, laisse transparaître l'égocentrisme du personnage qui, se plaçant au-dessus de la mêlée de ceux qui n'auraient pas su prendre

29 Ibid., p. 283.

30 Ibid., p. 282.

31 Ibid., p. 286.

32 Ibid., p. 281.

33 Ibid.

34 Ibid., p. 279.

35 Ibid., p. 287. 
à temps les décisions qui s'imposaient, attend des dirigeants du monde entier qu'ils se rangent derrière lui et lui laissent carte blanche pour empêcher cette catastrophe climatique dont les conséquences pourraient être comparables à l'extinction des dinosaures $^{(36)}$.

Par la suite, les craintes suscitées par ce personnage mégalomane seront plus que vérifiées lorsque sa proposition aventureuse de faire exploser une bombe atomique au cœur d'un volcan dans le but de provoquer un épais nuage de fumée capable de réduire le rayonnement solaire sur la planète s'avérera en réalité être le résultat d'une manipulation du conseil de crise mis en place autour de Milett par le professeur Eisele. En effet, pour des raisons de vengeance personnelle et de calcul financier, ce dernier cherche par tous les moyens à nuire aux intérêts économiques de la Chine et à condamner à l'échec le NASP, un projet de gigantesque centrale photovoltaïque africaine supposée alimenter en électricité l'Europe tout entière, au profit du projet concurrent du Northern Wind Project, qui mise quant à lui sur l'énergie éolienne et rapporterait des milliards à son entreprise Solunia, dont on apprend qu'elle finance à elle seule une part considérable des activités de l'IICO ${ }^{(37)}$.

Le troisième type de discours en lien avec la catastrophe est celui qui est tenu par le Gaia Camp de Diego Garcia, un groupe de résistance souterraine anticapitaliste vivant à l'écart de la société au fin fond du Mecklembourg, où ses membres s'adonnent essentiellement à l'élevage de poules et de moutons et tentent d'agir sur les consciences par le biais de clips vidéo postés sur YouTube. Suite à un mystérieux attentat à l'hôtel New York de Rotterdam laissant Mavie et Thilo Beck sans connaissance, ce dernier est hébergé dans la communauté par sa sœur, qui profite de la convalescence du climatologue pour récupérer les données présentes sur son ordinateur portable (les fameuses prévisions de Prometheus), ce qui offre aux Gaia Camps la possibilité d'être les premiers à annoncer au monde l'apocalypse imminente. La vidéo de propagande qu'ils en tirent, Save our Souls, véritable «clip d'horreur réalisé dans les règles de l'art de la communication et de la présentation» ${ }^{(38)}$, brille d'un côté par sa remarquable technicité et son aptitude à faire passer son message conspirationniste en se passant de mots, sur la seule base d'images suffisamment explicites pour être comprises par les hommes de tous les pays; de l'autre, mue par une volonté de faire éclater au grand jour les ficelles de l'ordre mondial, elle bascule littéralement dans l'amalgame et le ton accusateur d'une théorie du complot confuse et infondée:

«La musique demeurait menaçante tout du long, les premières images préparaient mentalement à la théorie du complot qui allait suivre. Des images aériennes de l’IICO, présenté sans autre forme de procès comme un centre de recherche secret du pentagone, [...] une série d'abréviations traversant l'espace vide, CIA, NSA, Mossad, MI6, les habituels suspects. Ensuite, le clip se composait essentiellement de chiffres et de captures d'écran, les captures d'écran de Beck [...]. La fin du clip était constituée d'un montage bigarré de formules, de visages et de courtes citations [...]. Les formules suggéraient simplement de manière sous-jacente que les scientifiques venaient de prouver quelque chose que d'autres avaient prédit depuis longtemps. Car les visages et les citations qui accompagnaient les

\footnotetext{
36 Ibid., p. 285.

37 Ibid., p. 388-392.

38 Ibid., p. 238.
} 
images d'inondation et de sécheresse étaient celles de prêtres mayas, de Nostradamus et, pour couronner le tout, d'Al Gore. Comme s'il était là pour soutenir le message: C'est la fin du monde ${ }^{(39)}$.

Rien, dans la narration, ne permet de remettre en question la sincérité de l'engagement du Gaia Camp, dont le rôle inestimable par rapport au dénouement de l'intrigue contribue à renforcer le statut héroïque. En dévoilant au monde entier le stratagème du professeur Eisele, ils évitent in extremis la réalisation de son plan machiavélique (faire exploser un volcan pour «vacciner la stratosphère» à l'aide du dioxyde de soufre contre son propre réchauffement, et par la même occasion mettre un terme aux projets de construction d'une gigantesque centrale photovoltaïque et nuire aux intérêts de la Chine). Néanmoins, outre les amalgames et les théories douteuses dont il se nourrit, leur discours sur la catastrophe et leur stratégie d'action se trouvent doublement décrédibilisés: d'une part, leur volonté d'être les premiers à annoncer le «scoop » et la teneur de leur message révèlent clairement leur refus délibéré de réfléchir aux conséquences concrètes de leurs actions en termes de retombées humaines, même lorsque plusieurs acteurs les mettent en garde contre l'hécatombe que pourrait provoquer une panique de masse; d'autre part, leur stratégie clandestine s'avère très facilement détournable au profit d'intérêts contraires lorsque le camp se trouve pris d'assaut par les hommes de Eisele, de manière à pouvoir exploiter leur matériel informatique, usurper leur identité et, ainsi, manipuler à distance la multitude de ceux qui considèrent la propagande des activistes comme une des rares sources fiables d'information.

En dehors de cette pluralité de discours sur la catastrophe, décrédibilisés les uns après les autres, au point qu'il est impossible de tirer du roman un message politique clair, celle-ci joue au sein de la narration le rôle de théâtre de l'action dramatique. Imminente puis en cours, la crise qu'elle représente crée une tension permanente qui pousse constamment les personnages à réagir dans l'urgence pour tenter de sauver ce qui leur tient le plus à cœur. Dès la première partie de l'ouvrage, la découverte de la catastrophe imminente constitue l'élément déclencheur du thriller, puisqu'elle met l'héroïne en danger et provoque l'assassinat (dissimulé en accident) de sa meilleure amie, la journaliste Helen, alors qu'elle était sur le point de faire éclater le scandale. Par la suite, le désastre climatique n'est que très rarement décrit pour lui-même. L'inondation de Hambourg, le blocage progressif de toutes les infrastructures de la ville portuaire ainsi que les conséquences pour tous ceux qui tentent de fuir la région sont essentiellement évoqués lors d'entretiens téléphoniques entre Mavie et son père, qui a depuis de nombreuses années transformé sa maison en un véritable bunker de crise rempli de boîtes de conserve, ou bien entre Mavie et son ami Daniel, dont les explications avisées contribuent à alimenter l'inquiétude de l'héroïne et la tension du lecteur.

«S'il continuait à pleuvoir et que le niveau de l'Elbe dépassait les sept mètres, alors à un moment donné tout sera fichu, en un rien de temps. Daniel dépeignait à Mavie les conséquences inévitables en les termes les plus sombres qu'on puisse imaginer - effondrement de l'alimentation électrique, effondrement de la communication et de toute la logistique. On ne manquerait pas d'eau, mais de tout le reste ${ }^{(40)}$.

39 Ibid., p. 238-239.

40 Ibid., p. 273. 
En dehors de quelques intrusions de la triste réalité par le biais des médias, la catastrophe humanitaire sert de toile de fond des décisions stratégiques lorsque les experts du monde sont réunis pour décider des mesures à prendre. De leur salle de conférence climatisée à Genève, climatologues, lobbyistes et prix Nobel sont rappelés par des écrans muraux à la triste réalité des échauffourées entre militaires et réfugiés bloqués aux frontières, des foules en fuite se battant pour obtenir un peu d'eau. Tandis qu'ils tentent de s'entendre sur une stratégie commune, ils ont en temps réel accès aux images $d^{\prime}$ 'u un monde [...] dans lequel minute après minute toujours plus d'hommes désespérés cherchent un chemin les conduisant vers des régions plus sûres et ne trouvent que la mort» ${ }^{(41)}$.

Enfin, dans la dernière partie du roman, intitulée «Styx», lors de l'épique opération de sauvetage des enfants de Philipp et de son ex-épouse par le trio composé de ce dernier, de Mavie et de son père, l'Elbe en crue dans laquelle pullulent de dangereuses méduses et sévissent des réfugiés africains devenus pirates devient un véritable fleuve des enfers ainsi que le terrifiant décor d'un roman d'aventure, dans lequel épreuves et obstacles font éclater au grand jour la bravoure et la noblesse d'âme des héros. Plus qu'à nourrir une réflexion sur les causes anthropiques et naturelles du changement climatique, la catastrophe crée les conditions d'une descente aux enfers individuelle et collective qui donne à certains personnages l'occasion de progresser dans leur quête et de dépasser leurs limites.

\section{Le climat pris en otage}

Dans son deuxième roman intitulé Sturm, l'auteur et entrepreneur munichois d'origine roumaine Uwe Laub traite, avec l'arme climatique et les tentatives militaires pour "contrôler le temps", d'un sujet dont Christian Chelebourg souligne à juste titre qu'il ne s'agit pas d'une idée nouvelle dans l'univers de la science-fiction puisque, dès 1959, l'auteur de bande dessinée belge Edgar P. Jacobs lui consacrait déjà un tome des aventures de Blake et Mortimer, dans lequel le colonel Olrik, chef d'un réseau d'espionnage étranger et ennemi juré du capitaine Blake et du professeur Mortimer, parvient à provoquer artificiellement en Europe occidentale toute une série de cataclysmes météorologiques ayant pour but de préparer le terrain à l'attaque d'une armée d'invasion ${ }^{(42)}$. Produit de la guerre froide et des angoisses nourries par la conquête de l'espace et l'arme nucléaire, la manipulation du climat constituerait une arme d'autant plus effrayante qu'elle serait difficile à détecter par ceux qui en feraient les frais:

«L'arme climatique apparaît d'autant plus efficace qu'elle réalise un paradoxe: redoutable par ses effets destructeurs et sa capacité à déstabiliser une zone, elle est quasiment indétectable en tant que moyen offensif puisque les phénomènes qu'elle occasionne peuvent toujours être attribués à une cause naturelle. Elle constitue un parangon de vecteur discret, aux antipodes des missiles nucléaires » ${ }^{(43)}$.

41 Ibid., p. 364.

42 S.O.S. Météores est la cinquième aventure et le huitième album de la série de bande dessinée Blake et Mortimer. Il paraît pour la première fois entre le 8 janvier 1958 et le 22 avril 1959 dans Le Journal de Tintin. Cf. Christian Chelebourg, Les écofictions. Mythologies de la fin du monde, Bruxelles, Les impressions nouvelles, 2012, p. 86. 
Cette caractéristique, qui fournit jusqu'à nos jours une matière propice à de nombreuses théories conspirationnistes invoquées pour expliquer des accidents météorologiques ${ }^{(44)}$, alimente pour une part considérable le suspense du roman d'Uwe Laub, dans lequel une accumulation de catastrophes climatiques, associée au meurtre d'un manager dans une affaire d'espionnage d'industriel, se révèle progressivement être l'œuvre d'un haut fonctionnaire chinois préparant une attaque inédite contre les États-Unis, supposée asseoir définitivement la suprématie mondiale de la Chine. Tout en s'inspirant du cinéma de la guerre froide pour élaborer son scénario, l'auteur fait habilement naviguer son thriller entre fiction et réalité. Dans la postface du roman comme sur son site Internet et dans le cadre de plusieurs interviews de presse, il indique notamment que l'idée maîtresse de l'ouvrage provient d'une étude de l'armée américaine publiée en 1996 et intitulée Le temps sera à nous en 2025 ${ }^{(45)}$, insiste sur les trois années de recherches qui ont été nécessaires à la rédaction de son livre et décrit de manière très détaillée sa visite du National Hurricane Center de Miami ainsi que les échanges qu'il a pu avoir avec certains experts. Dans la rubrique «Hintergrundinfos» de sa page web, Laub liste toute une série d'événements (comme le projet Popeye lancé pendant la guerre du Vietnam, visant à prolonger la mousson pour ralentir la progression des Vietcongs, les mesures mises en œuvre par la Chine à l'automne 2009 qui ont provoqué une tempête de neige sur Pékin, laquelle paralysa la ville pendant trois jours, etc.) et révèle des expériences en cours comme le programme américain HAARP de recherche sur l'ionosphère, et dont la base située en Alaska est régulièrement dénoncée comme la cause possible de certaines anomalies météorologiques, la construction en Norvège depuis 2015 de la station EISCAT 3D, financée en grande partie par des fonds européens, et l'existence en Chine d'une autorité pour la modification du temps supposée embaucher près de 30000 fonctionnaires. La conclusion alarmiste que l'auteur tire de ces phénomènes laisse supposer que l'action de son roman pourrait n'être à ses yeux qu'un avant-goût de ce que nous réserve un avenir relativement proche:

«Cela fait bien longtemps que les manipulations du temps ne relèvent plus du fantasme. Elles constituent des faits avérés. Chaque année, des milliers d'interventions sur la circulation atmosphérique ont lieu à travers le monde. De plus, d'innombrables expériences sont menées dans ce domaine. Les intentions vont d'objectifs raisonnables (production de pluie pendant des périodes de sécheresse, évitement d'averses de grêle) à des volontés criminelles (tempêtes, pluie continue et sécheresse de plusieurs mois utilisées comme armes de guerre) [...]. Mais n'oublions pas! Il ne s'agit là que d'intentions. Ce qui est réellement provoqué par ces expériences pourrait s'avérer bien plus dangereux [...]. L'exemple type des mathématiques du chaos sont les phénomènes atmosphériques. Si l'on tente de manipuler le temps à un point donné, les effets secondaires à l'échelle de la planète sont imprévisibles [...]. Mais si même une simple intervention ne peut rester sans conséquence, alors que causera au niveau mondial une accumulation croissante de manipulations? " ${ }^{(46)}$.

44 Ibid. Chelebourg rappelle notamment que sur certains sites conspirationnistes les violentes tempêtes de décembre 1999 avaient été interprétées comme des représailles contre l'attitude récalcitrante de la France à l'égard de la mondialisation, tandis que certains avaient vu dans la sécheresse qui a frappé la Russie en 2010 le résultat d'une attaque météorologique. 
Le roman, dont l'action se déroule simultanément en de multiples points du globe, est dominé par une dialectique fondamentale entre impuissance du plus grand nombre face aux catastrophes atmosphériques et puissance vertigineuse de ceux qui manipulent le climat. Dès les premières pages du thriller, le lecteur se trouve plongé dans un univers d'épouvante qui n'est pas sans rappeler l'atmosphère des films catastrophes hollywoodiens des années 70 et $80^{(47)}$. Lors d'un match de football opposant le FC Bayern au Herta BSC au stade olympique de Berlin, une tornade se forme en l'espace de quelques minutes au-dessus du Maifeld et fait voler en éclats la tour du carillon, ce qui cause l'effondrement de la cloche de plus de 4 tonnes sur la Langemarck-Halle avant de dévaster le stade, provoquant ainsi la mort de centaines de spectateurs pour qui toute tentative de fuite s'avère vaine. La description détaillée du phénomène, vu à travers les yeux à la fois fascinés et abasourdis du journaliste et spécialiste du climat Daniel Bender, venu assister au match avec son ami Leif Gundarsson, fait ressortir toute l'horreur de la situation et la puissance des éléments déchaînés sans ménager les sensibilités:

«La tornade atteignit le stade en effleurant la colonne de gauche de la porte du marathon. Elle s'effrita comme si elle était construite en sable. Le béton et le mortier giclèrent dans toutes les directions. Des spectateurs tombèrent à terre, touchés par des éclats tranchants. Mais la véritable horreur ne faisait que commencer. La tornade s'empara des premiers individus. Ceux-ci se retrouvaient aspirés, tourbillonnaient en l'air avant d'être recrachés. Relâchés de plusieurs mètres de haut, ils s'effondraient au sol, où ils gisaient sans vie » ${ }^{(48)}$.

Dans les chapitres suivants, la même incompréhension et la même impuissance frappent les hommes, qui se retrouvent confrontés à des catastrophes tout aussi violentes que soudaines et imprévisibles. À Iakoutsk, en Sibérie centrale, dans la grande ville la plus froide du monde, les travailleurs des mines de diamants du géant Alrosa découvrent lors de leur remontée à la surface que les températures, qui ne dépassaient pas les $-40^{\circ} \mathrm{C}$ quelques heures auparavant, sont en train de dépasser les $10^{\circ} \mathrm{C}$ et que ce dégel accéléré, parfaitement anormal pour la saison, génère une boue qui est en train d'engloutir de nombreux bâtiments et quartiers ${ }^{(49)}$. En Australie méridionale, près du phare de Point Malcom qui surplombe le canal reliant le lac Alexandrina et le lac Albert, deux retraités constatent successivement une accumulation de libellules mortes, une baisse du niveau du lac d'au moins quinze mètres en une seule nuit, puis une coloration argentée de ce dernier, qui s'avère être un effet d'optique causé par des milliers de poissons morts. Face à cette vision d'horreur, Steve invoque le monstre Muldjewangk, une créature aquatique de la mythologie aborigène réputée exiger de temps à autre son lot de sacrifices, tandis que son ami Riley préfère voir dans ce désastre une punition de Dieu ou bien l'œuvre du démon ${ }^{(50)}$. Enfin, lors d'une fête de village à Bredenstedt près de Hanovre, Laura Wagner et son fils Robin sont surpris par une violente tempête de grêle que personne n'avait annoncée; blessé à la tête, le

47 Sur l'histoire de ce genre, lire Stephen Keane, Disaster Movies: The Cinema of Catastrophe, Londres, Wallflower Press, 2006.

48 U. LAUB, Sturm (note 5), p. 14 (toutes les traductions sont nôtres).

49 Ibid., p. 16-23.

50 Ibid., p. 33-37. 
jeune garçon est conduit d'urgence à l'hôpital, où le personnel soignant doit gérer un nombre record de blessures graves liées aux intempéries. Et enfin, alors qu'elle se rend le lendemain au travail dans l'entreprise Andra AG, spécialisée dans les techniques de haute fréquence, Laura se trouve confrontée à une violente tempête de neige qui, en l'espace de quelques heures, paralyse toute la région. Contrainte de quitter son véhicule suite à un accident, Laura est sauvée de l'hypothermie par Daniel Bender et Leif Gundarsson, qui la conduisent au siège d'Andra, où les trois personnages se trouvent durablement bloqués pendant plusieurs jours par le blizzard qui sévit et une interdiction formelle de circuler émise par les autorités.

À ces scènes d'impuissance, dépeintes avec un réalisme presque choquant et interprétées dans un premier temps comme un véritable dérèglement du climat mondial, s'opposent les agissements de deux groupes proches des hautes sphères du pouvoir, qui se livrent un combat sans merci et semblent pouvoir manier à leur guise les éléments naturels. D’un côté, le haut-fonctionnaire du ministère chinois de la Sécurité publique, Huang Zhen, aidé par son cousin Xian Wang-Mei et son allié, le richissime noble britannique Charles St. Adams, contemple du haut de sa tour de contrôle insonorisée les 3600 antennes de l'installation de Heilongjiang, appelée le «dragon noir». Alors qu'il porte sur son kimono en soie les insignes impériaux, il s'apprête à rendre justice à son peuple en infligeant à son principal rival la plus grande leçon de son histoire:

«Zhen sursauta et indiqua de sa main tendue la salle de contrôle qui était en dessous d'eux:

“[...] La militarisation croissante que les États-Unis mettent en place devant chez nous constitue une atteinte à l'hégémonie chinoise dans l'espace pacifique. [...] C’est pourquoi nous devons démontrer notre pouvoir [...]. Ce pays ne comprend aucun autre langage. [...] Le dragon noir va rappeler à cette police planétaire suffisante qu'il existe des puissances plus fortes que la main humaine" "(51).

D’un autre côté, un conseil de crise occidental sous domination des États-Unis, doté d'une arme technologique encore plus puissante que celle des Chinois, l'installation HAARP 2 de 50000 antennes, orchestre dans l'urgence sa contre-attaque, tandis qu'Emily, monstrueuse tornade lancée par les Chinois, s'approche dangereusement des côtes américaines. Composé de spécialistes de la simulation météorologique, mais aussi du ministère américain de la Défense, de représentants de l'Agence américaine d'observation océanique et atmosphérique, de la NASA et de la CIA, le conseil se laisse en partie guider par les conseils de Daniel Bender, kidnappé pour l'occasion, dont on apprend qu'il est l'auteur d'une thèse de doctorat jadis refusée et qui a posé les bases théoriques permettant la construction des installations chinoise et américaine de manipulation du climat.

Prenant littéralement le climat mondial en otage, et avec lui l'existence de plusieurs centaines de millions d'individus à travers le monde, le bras de fer qui s'ensuit montre de quelle manière les forces de la nature jusque-là les plus indomptables peuvent être mises au service d'intérêts impérialistes. Ainsi, la description du combat et des catastrophes qui en découlent dévoile une des fonctions fondamentales de ce type de thriller climatique, déjà mise en évidence par Christian Chelebourg: grâce à l'écofiction, l'homme moderne n'est pas seulement mis en face d'une des pires évolutions possibles 
de son destin; il assouvit par la même occasion, le temps d'une lecture, son aspiration fondamentale à prendre la place de Zeus. «Commander au temps, c'est se substituer à Dieu lui-même, s'approprier l'expression de sa vindicte» ${ }^{(52)}$. Par-delà la mise en scène des dangers qui émanent de telles expériences, l'auteur nourrit à travers son œuvre une fascination propre à nos sociétés, en «clam[ant] notre force, aussi bien celle qui ravage l'environnement que celle qui serait capable de le sauver» ${ }^{(53)}$ : "L'alarmisme écofictionnel flatte le sentiment de grandeur qui habite les sociétés industrialisées. Il nous fait aimer le destin que nous nous sommes tracé en nous donnant l'impression que nous l'avons mené à son terme » ${ }^{(54)}$.

\section{Bilan}

Par son recours au cliché, sa volonté de tenir le lecteur en haleine et sa peinture quasi cinématographique des catastrophes, le thriller climatique ne laisse aucun doute sur sa fonction première, qui est de s'assurer des succès de librairie en divertissant un certain public en quête de suspense, de sensations fortes et de vulgarisation scientifique. Audelà de cette volonté fondamentale non dissimulée, le choix des grands enjeux de notre époque que sont la thématique environnementale et le positionnement de l'humanité face aux bouleversements atmosphériques qui nous guettent induit inévitablement un désir de faire réfléchir, même si la démarche d'auteurs comme Uwe Laub et Sven Böttcher ne peut à proprement parler être qualifiée de démonstrative ou de didactique. Frank Schätzing, l'auteur de Der Schwarm qui a largement servi de modèle à nos deux auteurs, exprime cette attitude de manière fort crue et sans équivoque dans l'une des multiples interviews qu'il a données à la presse allemande:

«J'ai un profond dégoût pour toute forme de messianisme. En réalité, ce que je recherche, c'est à me divertir moi et à divertir les gens. Ceci dit, je suis fasciné par les sujets dont la mise en œuvre ne réduit pas la pensée mais la sollicite. Tous les aspects en lien avec ces thèmes qui me passent par la tête, je les présente au lecteur comme au comptoir du rayon charcuterie. Il peut se servir ou choisir de ne pas le faire ${ }^{(55)}$.

Conformément à ce principe, les auteurs de ce genre émergent ne se reconnaissent pas comme engagés en faveur de la cause écologique; ils se défendent clairement de soutenir, à travers leurs romans, des positions politiques, idéologiques ou éthiques particulières. Ils tendent plutôt à confronter les lecteurs à différents points de vue, qui se retrouvent régulièrement relativisés voire discrédités par l'attitude des personnages plus ou moins caricaturaux qui les représentent. Conformément aux codes du thriller, le seul message clair qui semble pouvoir être tiré de leur représentation des catastrophes et de l'action mouvementée et trépidante de ces ouvrages est que, dans notre monde, on ne sait souvent pas qui «tire les ficelles» de la réalité; le climat, aussi bien que la cause écologique, tend à être manipulé secrètement au nom d'intérêts économiques,

C. Chelebourg, Les écofictions (note 42), p. 84-85.

Ibid., p. 228.

Ibid.

55

Interview de Frank Schätzing: "Wir neigen zum Plündern», Der Tagesspiegel, 08.10.2010, www. tagesspiegel.de/gesellschaft/medien/interview-wir-neigen-zum-pluendern/1952482.html (consulté le 21.02.2020). 
financiers et impérialistes. Plutôt que de solliciter un engagement de type écologiste qui pousserait chacun à prendre ses responsabilités, ces auteurs cultivent certaines affinités avec les théories complotistes. Enfin, à un niveau plus général, le thriller climatique apparaît comme un digne héritier de certains grands classiques de la science-fiction: tout en mettant en garde contre certaines évolutions, il cultive une certaine fascination pour les possibles de la science et conforte ainsi l'homme des sociétés industrialisées dans son sentiment de grandeur et de puissance.

\section{Résumé}

Avec la parution de romans comme Go! Die Ökodiktatur (1993) de Dirk C. Fleck puis de Der Schwarm (2004) de Frank Schätzing, le genre du thriller écologique de langue allemande a atteint, notamment grâce à la place centrale qu'il accorde aux catastrophes environnementales, de remarquables succès de librairie. Sa variante particulièrement actuelle, le thriller climatique, cherche à la fois à choquer et à divertir, tout en accordant une place de choix à la transmission de connaissances sur le changement climatique. $\grave{A}$ travers l'analyse de la représentation des catastrophes climatiques dans deux romans récents, Prophezeiung (2011) de Sven Böttcher et Sturm (2017) de Uwe Laub, nous montrons que, par-delà une volonté manifeste de faire réfléchir, ces thrillers, dans lesquels l'influence de blockbusters américains et de théories complotistes est largement perceptible, ne sont guère les porteurs d'un message politique clair en faveur de la lutte contre le réchauffement de la planète. À l'instar de certains grands classiques de la sciencefiction, ils tendent à conforter la société contemporaine dans son sentiment de grandeur et de puissance.

\section{Zusammenfassung}

Mit der Veröffentlichung von Romanen wie Go! Die Ökodiktatur (1993) von Dirk C. Fleck und Der Schwarm (2004) von Frank Schätzing hat der deutschsprachige Ökothriller nicht zuletzt wegen der zentralen Bedeutung, die er Umweltkatastrophen beimisst, bemerkenswerte Buchhandelserfolge erzielt. Seine besonders aktuelle Variante, der Klimathriller, will sowohl schockieren als auch unterhalten und zugleich über den Klimawandel Wissen vermitteln. Durch eine Analyse der Darstellung von Klimakatastrophen in zwei aktuellen Romanen, Prophezeiung (2011) von Sven Böttcher und Sturm (2017) von Uwe Laub, soll gezeigt werden, dass diese Thriller, in denen der Einfluss von amerikanischen Blockbustern und von Verschwörungstheorien deutlich spürbar ist, zwar offensichtlich zum Nachdenken anregen, doch kaum als Träger einer klaren politischen Botschaft zugunsten des Kampfes gegen die globale Erwärmung betrachtet werden können. Wie einige der großen Klassiker der Science-Fiction tendieren sie dazu, das Gefühl der heutigen Gesellschaft für Größe und Macht zu verstärken.

\footnotetext{
Abstract

With the publication of novels such as Go! Die Ökodiktatur (1993) by Dirk C. Fleck and Der Schwarm (2004) by Frank Schätzing, the German ecological thriller has become a best-selling genre, especially thanks to the special prominence it gives to environmental disasters. Its latest variant, the climate thriller, aims to shock as well as to entertain;
} 
simultaneously, it emphasizes the importance of educating the reader on climate change. By analyzing the way natural disasters are represented in two recent novels, Prophezeiung (2011) by Sven Böttcher and Sturm (2017) by Uwe Laub, it is intended to show that no matter how thought-provoking these thrillers may be and in spite of the perfectly obvious influence of American blockbusters and conspiracy theories, they don't send out a clear political message in favour of the fight against global warming. Like some of the great classics of science fiction, they tend to strengthen the sense of greatness and power of our contemporary society. 